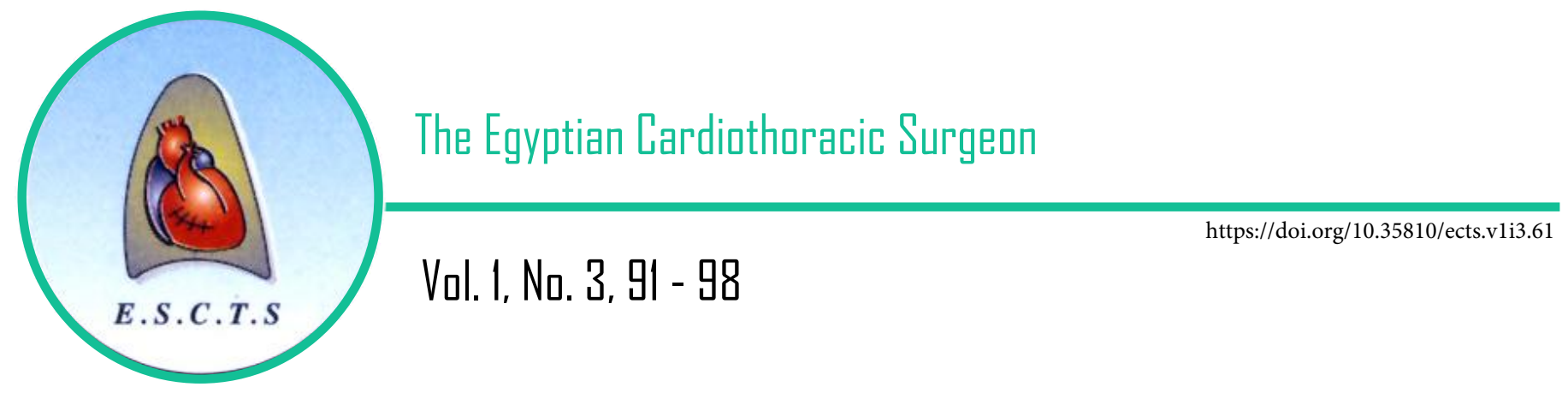

Original Article

\title{
The use of cardiopulmonary bypass in the extraction of intracardiac foreign bodies
}

\section{Mohamed Mahmoud Mostafa}

Cardiothoracic Surgery Department, Faculty of Medicine, Assiut University Heart Hospital, Assiut, Egypt

\section{Abstract}

Background: Intracardiac foreign bodies (FBs) are uncommon and have diverse presentations. The objectives of this study were to assess the types and presentation of intracardiac FBs and to evaluate the use of cardiopulmonary bypass (CPB) in their extraction.

Methods: A retrospective descriptive study was carried out on 12 patients with a history or radiological evidence of a foreign body in the heart or the great vessels who were admitted between 2013 and 2018. Sternotomy was performed in 8 patients and left anterior thoracotomy in 4 patients. CPB was used in 4 patients with cardioplegic cardiac arrest. Aorto-bicaval cannulation was performed in 3 patients and femero-femoral bypass in 1 patient.

Results: The mean age of our patients was $32.7 \pm 21.7$ years (range 2-62 years), six were males. Six different intracardiac FBs were reported including retained bullets $(n=3)$, migrated catheter piece $(n=3)$, sewing needles $(n=3)$, displaced pacemaker lead $(n=1)$, circular saw $(n=1)$ and missed pigtail catheter after pericardiocentesis $(n=1)$. Recovery from cardiopulmonary bypass was smooth, and no hospital complications were reported. The mean duration of postoperative mechanical ventilation in all sternotomy patients was $7.8 \pm 6.7$ hours ( $5 \pm 2.1$ in CPB patients and 10.7 \pm 8.9 in non- CPB). The duration of hospital stay in $\mathrm{CPB}$ cases vs. non-CPB was $(5.5 \pm 1.3$ vs. $5.7 \pm 0.9$ days). No postoperative wound infection nor sternal dehiscence were reported. One baby who had lateral thoracotomy died on the fifth postoperative day because of severe gastroenteritis. No residual pericardial or pleural collection were reported in 6 months follow-up period.

Conclusions: Retrieval of intracardiac FBs can be performed safely with low morbidity and mortality. The use of CPB did not increase morbidity or mortality. Removal of all types of intracardiac FBs is recommended to avoid complications

\section{KEYWORDS}

Cardiopulmonary

bypass; Intracardiac foreign bodies;

Retained bullets

\section{Article History}

Submitted: 30 Mar 2019

Revised 1: 10 Apr 2019

Revised 2: 13 May 2019

Accepted: 14 May 2019

Published: 1 July 2019 


\section{Introduction}

Intracardiac foreign bodies (FBs) are serious traumatic injuries with high mortality and are technically challenging [1]. Intracardiac FBs have several complications, and the prompt diagnosis and surgical intervention are required to prevent adverse consequences [2]. Several types of FBs were retrieved from the heart such as bullets, shrapnel, catheters and migrating wires from peripheral vessels [3]. The intracardiac FBs can occur after a surgical procedure such as acrylic cement embolism in percutaneous vertebroplasty, and sternal bars fragments after surgery for pectus deformity $[4,5]$. FBs can embolize during stent placement for Budd-Karri syndrome, and portosystemic shunts and needles can reach the heart through several ways [5].

Presentation varies from life-threatening bleeding to accidentally discovered FBs. The frequent clinical symptoms are chest discomfort and shortness of breath. However, patients can be asymptomatic for several years and present with atypical or a misleading history. Patients can present with FBs complications such as infective endocarditis, repeated pericardial effusions, pericarditis, constrictive pericarditis, perforation and tamponade, valve dysfunction, and arrhythmia $[6,7]$. Thus, rapid retrieval of cardiac $\mathrm{FBs}$ is recommended.

The precise localization of the foreign body is essential before surgical intervention. Chest X-ray and CT scans are crucial to identify the site, size, and numbers of retained foreign bodies, particularly when the history is uncertain [8]. Transthoracic echocardiography (TTE) provides information on the dimension, site, motion, and connection of foreign bodies, in addition to the cardiac function. It has high sensitivity and is the most useful diagnostic tool [9].

The objectives of this study were to assess the types and presentation of intracardiac FBs and to evaluate the use of cardiopulmonary bypass (CPB) in their extraction.

\section{Patients and Methods:}

This is a retrospective descriptive study including 12 patients with intracardiac FBs, presented between 2013 and 2018 and were treated surgically. Sternotomy was performed in 8 patients and left anterior thoracotomy in 4 patients. CPB was used in 4 patients with antegrade cold cardioplegic arrest, aorto-bicaval cannulation was performed in 3 patients and femero-femoral bypass in 1 patient. Institutional Ethical Committee approval to perform the study was obtained prior to data collection.

\section{Bullets in the heart cavities ( $n=3$ )}

Patient 1: A 55-year-old man presented with a gunshot in the chest. Chest $x$-ray showed retained bullet in the cardiac shadow (Figure 1A), and multi-slice chest CT showed a bullet in the right ventricle (RV) (Figure 1B). TTE revealed minimal pericardial effusion. A median sternotomy was done with the use of CPB and cold cardioplegic arrest. The bullet was extracted through a right ventricular incision (Figure 1C) which was repaired by interrupted polypropylene $4 / 0$ on Teflon pledget.

Patient 2: A 32-year-old female had a gunshot inlet in the epigastrium. A chest $x$-ray showed bullet in the RV and gas under the diaphragm. TTE revealed massive pericardial effusion and tamponade. Emergency median sternotomy and aorto-bicaval cannulation were performed. The bullet transfixed the diaphragm and the inferior surface of the heart and embedded in the RV near the left anterior descending (LAD) coronary artery (Figure 2). The inferior surface of the heart was repaired with polypropylene $4 / 0$ on Teflon pledget, and the diaphragmatic tear was closed. The RV was opened anteriorly to extract the bullet (Figure 3) then repaired with polypropylene $4 / 0$ on Teflon pledget with great care to the course of the LAD. The patient had a concomitant tear in the transverse colon, and a colostomy was done which was closed after two weeks.

Patient 3: A 50-year-old man had a bullet in the right ventricle which was extracted with the same technique as patient 1 .

\section{Needles in the heart $(n=3)$}

Patient 4: A 2.5-year-old boy had a penetrating needle during movements in the cradle. Chest $x-$ ray revealed the shadow of the needle in the RV. A left anterior thoracotomy at the fourth intercostal space was performed. The needle was partially covered by fibrin and partially embedded in the ventricle. Needle extraction was done, and 

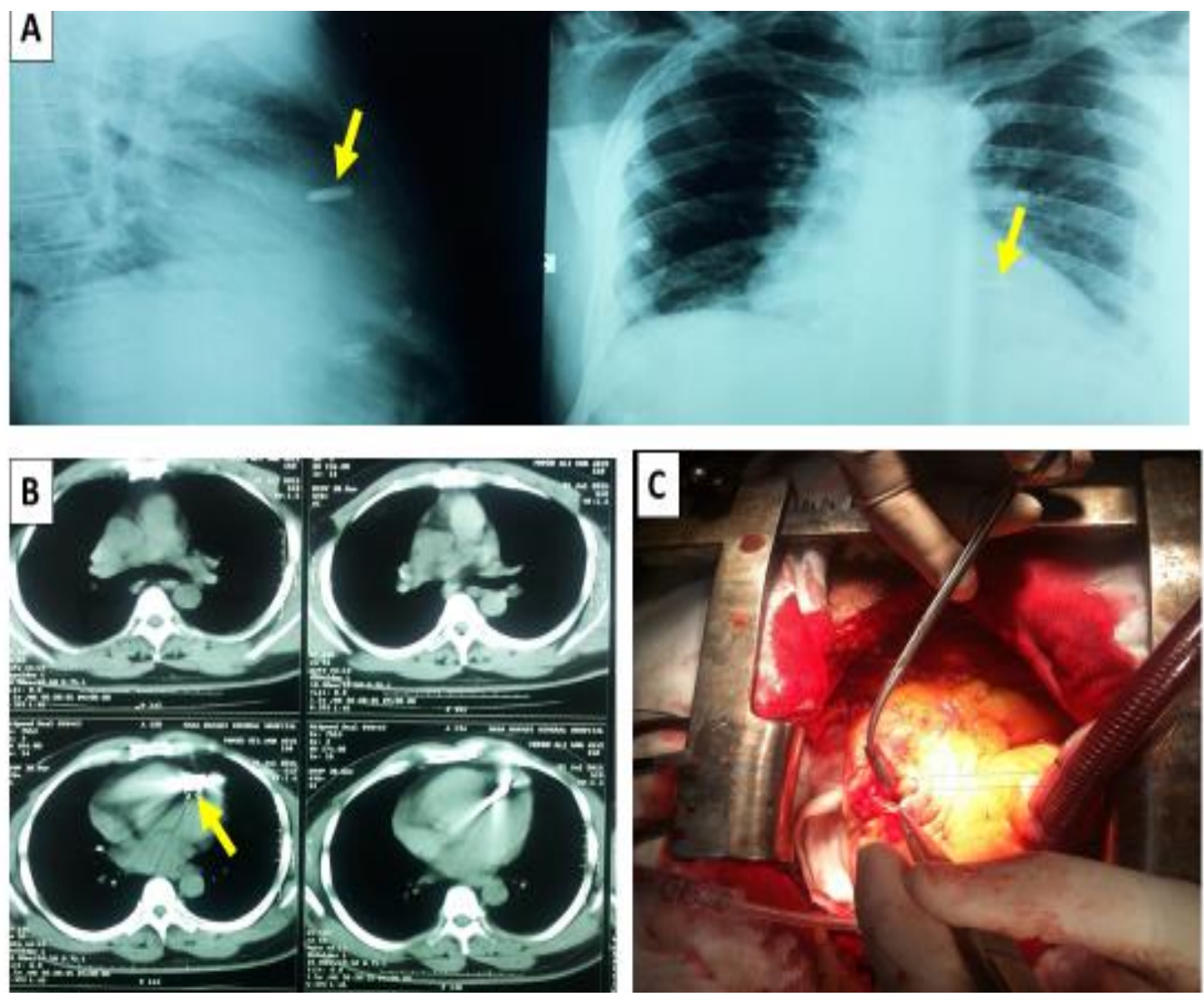

Figure 1: A-Chest $x$-ray posteroanterior and lateral views are showing retained bullet (firearm injury) in the cardiac shadow in the area of the right ventricle (arrowhead). B-Chest CT revealing retained bullet in the right ventricle just below the right ventricular outflow tract. C-Intraoperative photo showing the identification and removal of the retained bullet from the right ventricle with cardiopulmonary bypass and cardioplegic arrest.
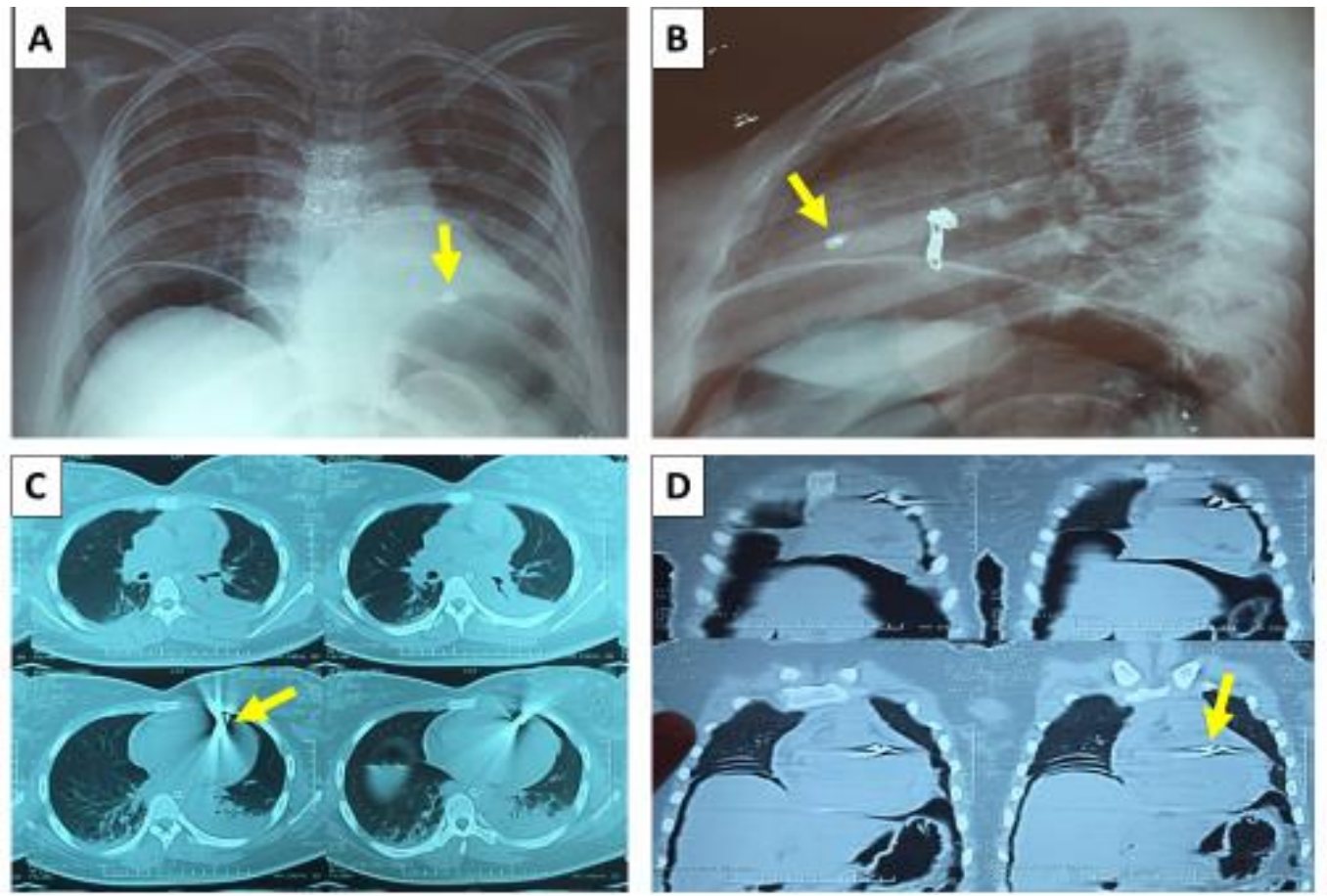

Figure 2: Chest $x$-ray posteroanterior and lateral views showing retained bullet in the cardiac shadow (arrowhead) and gas under the diaphragm, the inlet of the bullet was in the epigastric region and perforated the transverse colon, diaphragm and retained in the heart (A and $B)$. Chest $C T$ image revealing retained bullet in the right ventricle and left-sided hemothorax. (C) and gas under the diaphragm (D). 

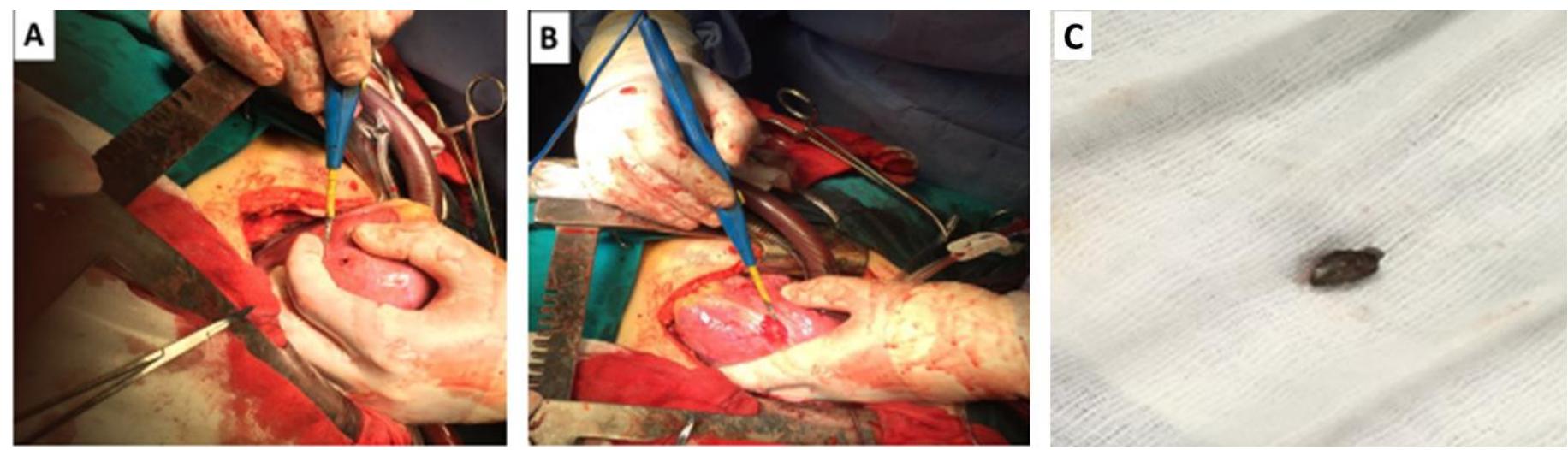

Figure 3: A photograph during surgery is showing firearm injury bullet that transfixed the inferior surface of the heart and retained in the wall of the right ventricle just near the LAD (arrowed by the tip of the diathermy) ( $A$ and $B$ ). The bullet after extraction (C).

burse polypropylene 4/0 was taken around the exit of the needle.

Patient 5: A 2-year-old girl had a partially penetrating needle in the right ventricle which was extracted through a left thoracotomy.

Patient 6: A 2.5-year-old boy had a needle penetrating the RV which was removed successfully. The patient had severe gastroenteritis, dehydration, and febrile convulsions and died on the fifth day postoperative.

\section{Retained catheter fragments $(n=3)$}

Patient 7: A 62-year-old man had a left-sided flail chest with difficult weaning from the mechanical ventilator after a car accident. Surgical fixation of fractured ribs was done by titanium plates and screws; postoperatively the patient had recurrent paroxysmal tachyarrhythmia. Chest $x-$ ray showed retained central venous line (CVP) wire in the region of superior vena cava (SVC), right atrium and the inferior vena cava (IVC) (Figure 4). A median sternotomy was done, and the wire was extracted through a right atrial incision without CPB.

Patient 8: A 35-year-old female was admitted to ICU with preeclampsia, and during the introduction of the CVP, the wire migrated to the heart. The patient had median sternotomy, and the wire was extracted through a right atriotomy without CPB.

Patient 9: A 22-year-old man had atrial septal defect closure. Postoperatively, the CVP cannot be removed. During Reoperation, the CVP was attached to SVC sutures and was removed successfully after the release of the sutures.
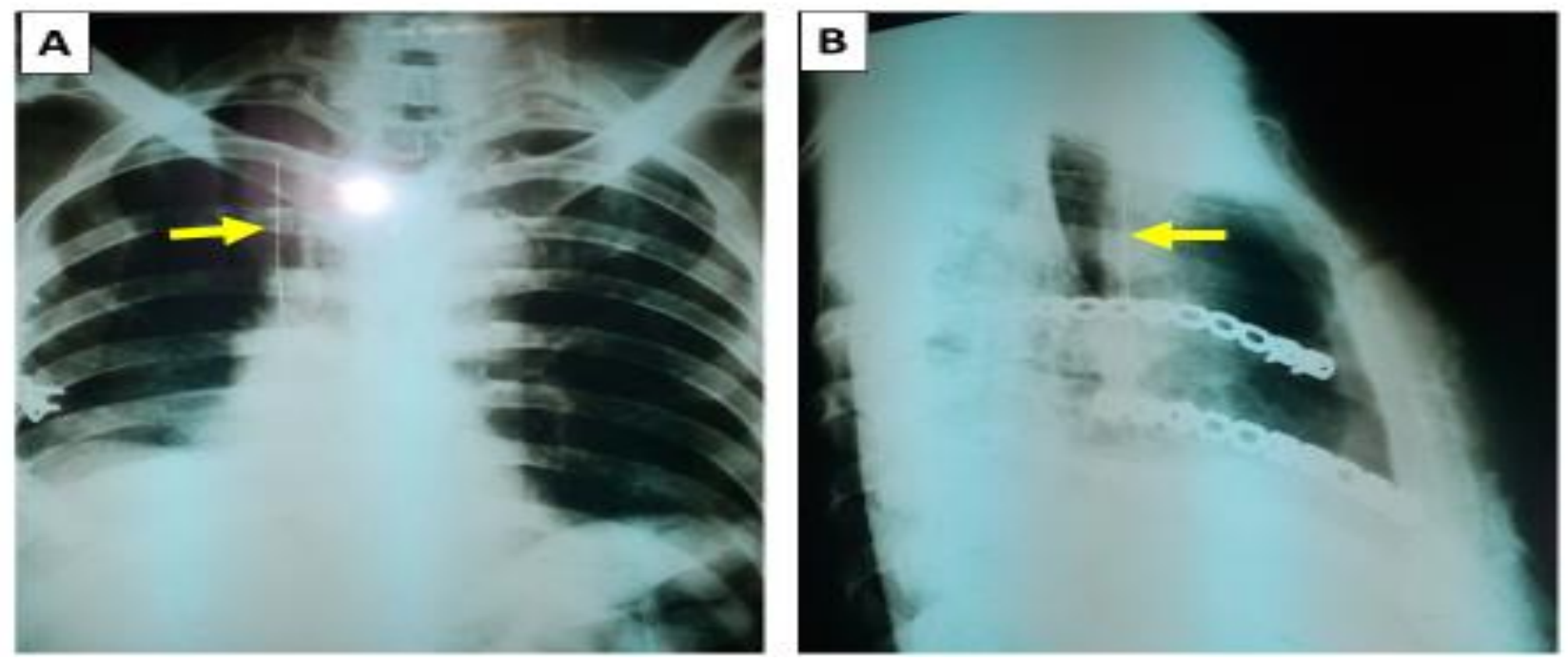

Figure 4: Chest $x$-ray (posteroanterior $(A)$ and lateral $(B)$ views) with right side rib plating with plates and screws showing missed retained CVP wire in superior vena cava and the right atrium (arrowhead). 
Dislodgement of the pacemaker lead $(n=1)$

Patient 10: A 55-year-old female had a right ventricular tear during removal of the pacemaker lead which was retained in the RV. The patient presented with cardiac tamponade. CPB was instituted by femero-femoral bypass then a median sternotomy was done. Right atriotomy and removal of the dislodged lead through the tricuspid valve were accomplished. Repair of the right ventricular tear by interrupted polypropylene $4 / 0$ on Teflon pledget and closure of the right atriotomy were completed.

\section{Fragment of a circular saw in the right ventricle $(n=1)$}

Patient 11: A 45-year-old man had a fragment of a circular saw in the RV, which penetrated while the patient was working and resulted in cardiac tamponade. A median sternotomy was performed, and CPB was instituted. The fragment was removed, and the RV was repaired.

\section{Fragment of pigtail catheter in the right ventricle} after pericardiocentesis $(n=1)$

Patient 12: A 30-year-old female had systemic lupus erythematosus presented with massive pericardial effusion. Pigtail was inserted under echo-guidance for pericardiocentesis. During its removal on the fourth day, the catheter was not removed completely. Limited left-sided anterior thoracotomy on the fifth intercostal space was done. The embedded tip of the catheter was found in the myocardium of the RV with evidence of pericarditis.

\section{Postoperative assessment:}

Operative mortality was defined as death in hospital or within 30 days after surgery. All patients were followed up for six months by chest $X$-ray and echocardiography to evaluate the pericardial or pleural collection.

\section{Results}

The mean age of our patients was $32.7 \pm 21.7$ years (range 2-62 years), six were males. Six different FBs were reported in the 12 patients (Table 1) including retained bullets $(n=3)$, migrated catheter piece from a peripheral vessel $(n=3)$, sewing needle $(n=3)$, displaced pacemaker lead $(n=1)$, circular saw $(n=1)$ and missed pigtail catheter after pericardiocentesis $(n=1)$. Surgical retrieval of the FBs was performed in all patients.

Sternotomy was done in 8 patients. CPB was used in 4 cases, 3 cases had aorto-bicaval cannulation, and one case had femero-femoral bypass. The mean duration of postoperative mechanical ventilation in patients who had sternotomy was $7.8 \pm 6.7$ hours and ranged from 5 to 24 hours ( $5 \pm 2.1$ in CPB patients and $10.7 \pm 8.9$ in patients who did not need (CPB). After removal of the mediastinal drains and intercostal tubes, the patients were transferred to the ward for 48 hours then discharged. Review after six months revealed proper wound healing and sternal union. Follow up imaging revealed no residual pericardial or pleural collection. The duration of hospital stay in CPB patients vs. non-CPB patients was $5.5 \pm 1.3$ vs. $5.7 \pm 0.9$ days respectively. Recovery from cardiopulmonary bypass was smooth with no postoperative complications.

Left anterior thoracotomy was done in 4 cases; the patients were transferred to the intermediate care unit and were observed for 24-48 hours postoperatively. No evidence of retained FB or effusions was found on follow up chest X-ray and echocardiography. One patient died in the fifth postoperative day in the left thoracotomy group because of severe gastroenteritis, dehydration, and febrile convulsions.

\section{Discussion}

Intracardiac FBs are rare but with high surgical risk and technically demanding. They require proper planning before surgery to accurately localize the $\mathrm{FBs}$ and achieve satisfactory results. The FBs can reach the heart by direct penetration or migration during other procedures [10]. There were only 40 cases of intracardiac sewing needle penetration described from 1967 to 2013. Patient with major psychiat-ric disorder represents $90 \%$ out of the whole number of patients presented in this series [5]. We found six different unusual FBs types including retained bullets, migrated catheter piece, lost needle in the baby's bed afterward piercing the baby's thorax, displaced pacemaker lead, circular saw in the right ventricle and missed pigtail catheter after pericardiocentesis.

Cardiac FBs may present with life-threatening emergency or discovered accidentally during the 


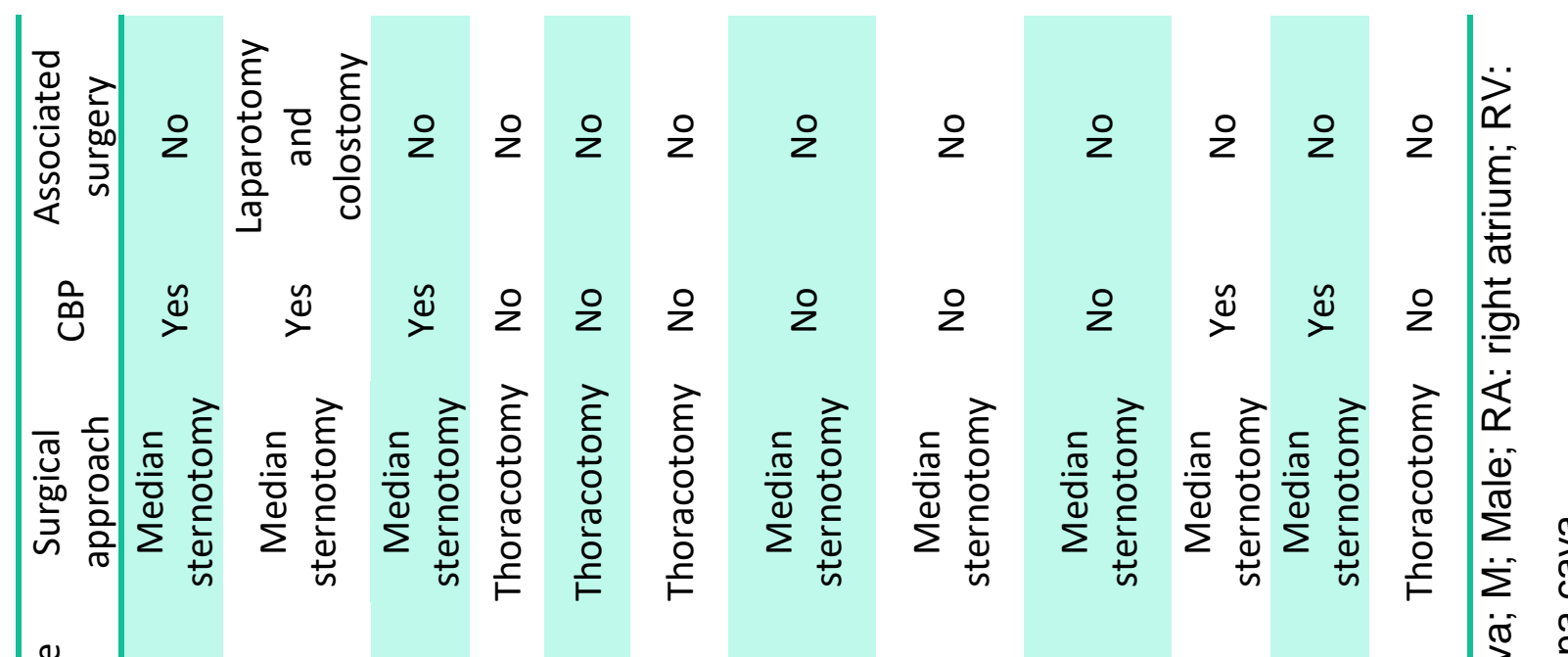

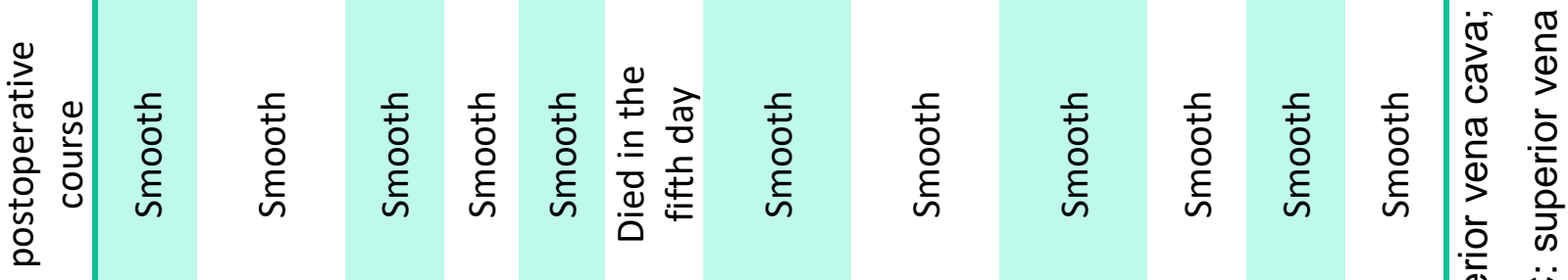

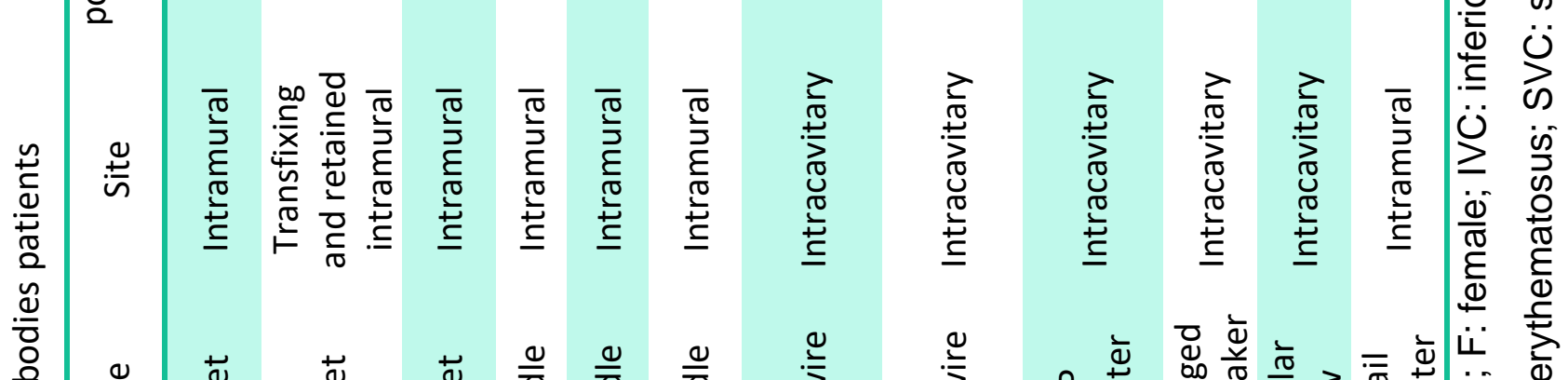

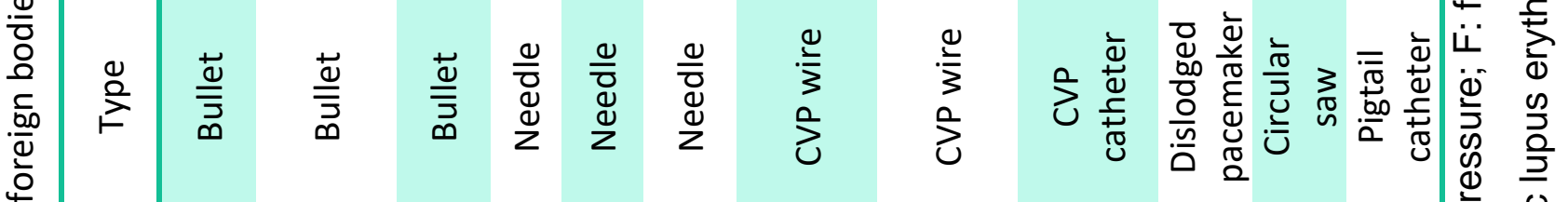
范

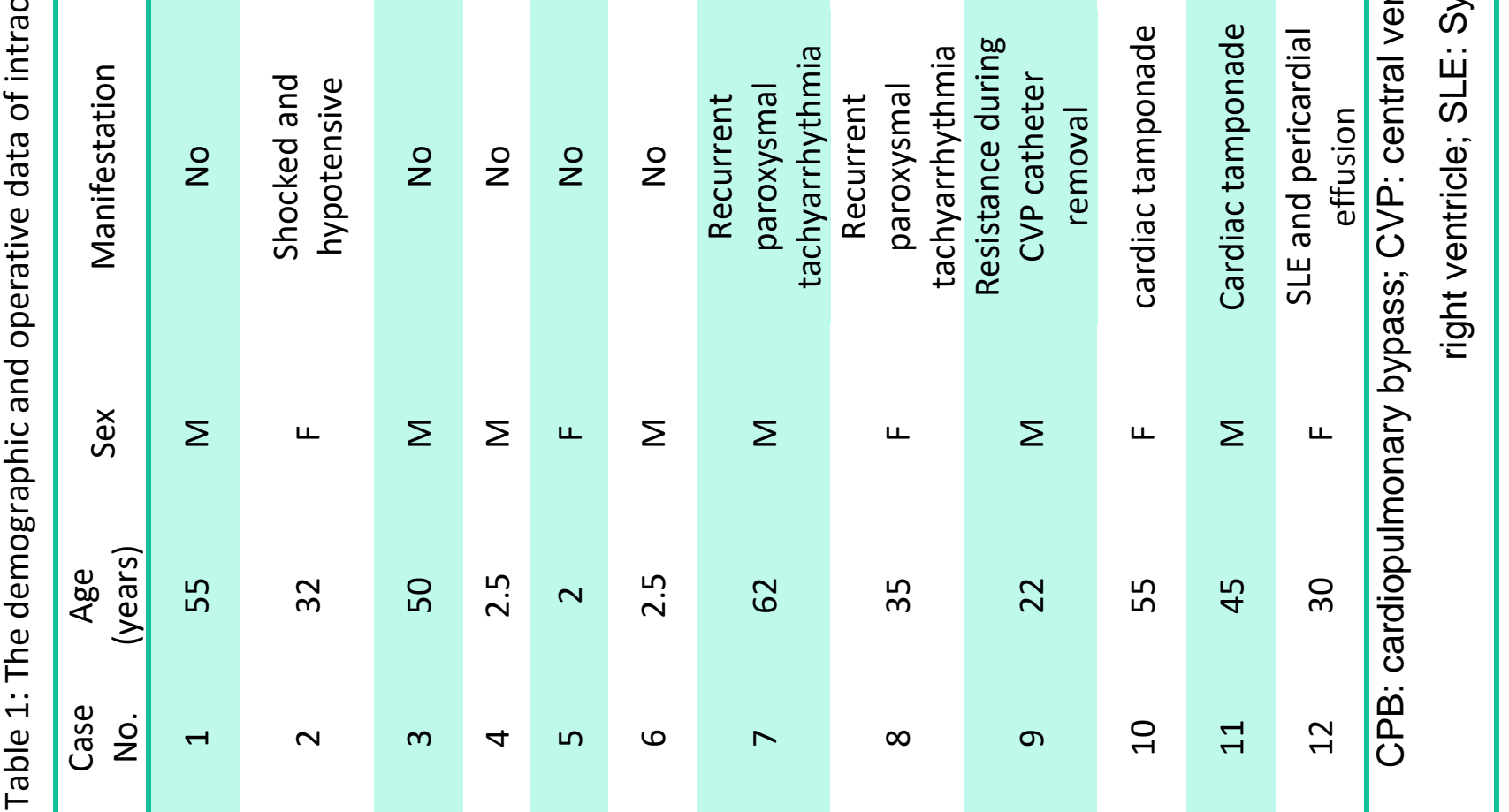


investigation for other conditions. The intracardiac FBs could manifest immediately after the injury with cardiac tamponade and/or hemorrhage. If the FBs did not injure the valves or septa, the patient could be asymptomatic at presentation. When diagnosed later, most cardiac foreign bodies have been trapped by fibrous tissue. In certain patients, they can develop symptoms several years after conservative management of cardiac foreign body [10, 11]. The most common manifestations are fever, pericarditis, and pericardial effusion, thrombi, arrhythmia, and neurotic manifestations, mostly anxiety. Fever might result from contamination of pericardium or endocardium or sepsis, particularly from infected fragments if the patient was conservatively treated [12].

Patient history, chest x-ray films, chest multislice CT scan, TTE, and cardiac fluoroscopy were helpful tools in the diagnosis and exact orientation of intracardiac FBs $[1,9,13]$. The principal goals of management of intracardiac FBs are saving the lives of the patients, removing the FBs, and preventing future complications. Therapeutic options including surgical removal and conservative treatments which should be tailored according to the patient requirements $[2,11,14]$. Complications of FBs favor early surgical intervention [15]. Even though, controversy continues in the literature around the suitable treatment of asymptomatic intracardiac FBs. Perrotta et al. found that in patients with sewing needle cardiac injuries, surgical intervention was preferable to conservative management [16].

Asymptomatic patients have definite hazards of late embolization, infection or erosion, and septic consequence from the retained pins and needles [2]. Therefore, most of the authors stated that any lag to remove the intracardiac FBs would lead to serious complications $[2,7,17]$. The present study is in agreement with that opinion to avoid further problems in delayed presentation. latrogenic foreign bodies have medicolegal consequences that occasionally can affect the choice between surgical or conservative treatment.

In the current series, the surgical approach was through median sternotomy in 8 patients (CPB was used in 4 of them) and left anterior thoracotomy in 4 patients. Surgical choices depend on the position of the foreign body, the cardiac chamber tangled, relationship to heart cavity and the requirement for CPB. The majority of cardiac wounds can be sutured with direct stitches without CPB and FBs that presented with a partially exposed portion outside the cardiac cavities could be approached without CPB [11].

Previous reports propose that a median sternotomy approach lead to reduced illness and decreased hospitalization in comparison to thoracotomy $[10,12]$. Several case reports have described the successful removal of intracardiac FBs using CPB $[5,9,11,12]$. Our results are in accordance with the above-mentioned findings to avoid bullet embolization and traumatic intracardiac defects, along with subsequent heart failure $[1,2,7]$. Percutaneous removal was reported with catheter fragments and should be performed as preliminary therapy, not prohibiting consequent surgical procedure [15,18,19].

CPB allows greater safety and upsurges the patient survival as it allows still bloodless field when the FBs can be retained in a heart cavity or transfixing the heart or have difficult location as near left anterior descending coronary artery as described in our patients. In addition, closure of cardiac defects caused by FBs penetration can be repaired with the use of CPB [1].

\section{Study limitations:}

The main limitation is the small sample size and retrospective nature of the study. However, this is a rare entity, and this study presents one of the largest series of intracardiac FBs.

\section{Conclusion}

Retrieval of intracardiac FBs can be performed safely with low morbidity and mortality. Preoperative imaging is essential for proper localization and planning surgical intervention. The use of CPB did not increase morbidity or mortality. Removal of all types of intracardiac FBs is recommended to avoid complications.

Conflict of interest: Authors declare no conflict of interest. 


\section{References}

1. Fu $\mathrm{H}-\mathrm{Y}$, Ting $\mathrm{M}$, Wang $\mathrm{Y}-\mathrm{C}, \mathrm{Yu} \mathrm{H}-\mathrm{Y}$. Intracardiac foreign body resulting from a transmediastinal gunshot mimics an extracardiac foreign body: An image presentation. Journal of the Formosan Medical Association. 2017;116(10):815-8.

2. Wang $X$, Zhao $X$, Du D, Xiang $X$. Management of metallic foreign bodies in the heart. Journal of cardiac surgery. 2012;27(6):704-6.

3. Mitsopoulos G, Hanna RF, Brejt SZ, et al. Retrieval of a Dislodged Catheter Using Combined Fluoroscopy and Intracardiac Echocardiography. Case reports in radiology. 2015;2015.

4. Tubbax H, Hendzel P, Sergeant P. Cardiac perforation after Kirschner wire migration. Acta Chirurgica Belgica. 1989;89(6):309-11.

5. Darmawan H. An Unusual Foreign Body in the Heart: A Case Report. Annals of Thoracic and Cardiovascular Surgery. 2018;24(4):205-7.

6. Inoue T, lemura J, Saga T. Delayed cardiac tamponade caused by self-inserted needles. The Canadian journal of cardiology. 2003;19(3):306-8.

7. Mandegar $\mathrm{MH}$, Ali Yousefnia $\mathrm{M}$, Rayatzadeh $\mathrm{H}$, Roshanali F. Intramyocardial sewing needle extracted one year after insertion. Interactive cardiovascular and thoracic surgery. 2006;5(6):742-3.

8. Yanar H, Aksoy M, Taviloglu K, Unal E, Kurtoglu M, Nisli K. Trans-sternal cardiac injury caused by a hooked needle. Emergency medicine journal. 2005;22(10):751-3.

9. Jiang C-L, Gu T-X, Wang C. Surgical treatment of posttraumatic foreign bodies in the heart or great vessels. Chinese medical journal. 2006;119(23):2018-20.

10. Dato GMA, Aidala E, Zattera GF. Foreign bodies in the heart: surgical or medical therapy? The Annals of thoracic surgery. 1999;68(1):291.

11. Dato GMA, Arslanian A, Di Marzio P, Filosso PL, Ruffini E. Posttraumatic and iatrogenic foreign bodies in the heart: report of fourteen cases and review of the literature. The Journal of thoracic and cardiovascular surgery. 2003;126(2):408-14.

12. LeMaire SA, Wall Jr MJ, Mattox KL. Needle embolus causing cardiac puncture and chronic constrictive pericarditis. The Annals of thoracic surgery. 1998;65(6):1786-7.

13. Nagy KK, Gilkey SH, Roberts RR, Fildes JJ, Barrett J. Computed tomography screens stable patients at risk for penetrating cardiac injury. Academic Emergency Medicine. 1996;3(11):1024-7.

14. Lundy JB, Johnson EK, Seery JM, Pham T, Frizzi $J D$, Chasen AB. Conservative management of retained cardiac missiles: case report and literature review. Journal of surgical education. 2009;66(4):228-35.

15. Pathak K, Yadav D. Percutaneous removal of intracardiac and intravascular foreign bodies. Medical Journal Armed Forces India. 2011;67(4):367-9.

16. Perrotta S, Perrotta A, Lentini S. In patients with cardiac injuries caused by sewing needles is the surgical approach the recommended treatment? Interactive cardiovascular and thoracic surgery. 2010;10(5):783-92.

17. Talwar S, Subramaniam KG, Subramanian A, Kothari SS, Kumar AS. Sewing needle in the heart. Asian Cardiovascular and Thoracic Annals. 2006;14(1):63-5.

18. Dakshinamurti S, Ducas J, Odim J. Retrieval of Silastic catheter fragment from heart in septic thromboembolism complicating aplastic anemia. The Canadian journal of cardiology. 1996;12(9):794-6.

19. Best IM. Transfemoral extraction of an intracardiac bullet embolus. The American surgeon. 2001;67(4):361. 